\title{
Exceptional $\mathcal{N}=3$ theories
}

\author{
Iñaki García-Etxebarria ${ }^{a}$ and Diego Regalado ${ }^{a, b}$ \\ ${ }^{a}$ Max Planck Institute for Physics, \\ Föhringer Ring 6, 80805 Munich, Germany \\ ${ }^{b}$ Institute for Theoretical Physics and Center for Extreme Matter and Emergent Phenomena, \\ Utrecht University, Leuvenlaan 4, 3584 CE Utrecht, The Netherlands \\ E-mail: inaki@mpp.mpg.de, regalado@mpp.mpg.de
}

ABSTRACT: We present a new construction of four dimensional $\mathcal{N}=3$ theories, given by M5 branes wrapping a $T^{2}$ in an M-theory U-fold background. The resulting setup generalizes the one used in the usual class $\mathcal{S}$ construction of four dimensional theories by using an extra discrete symmetry on the M5 worldvolume. Together with the M-theory U-fold description of $(0,2) E$-type six-dimensional SCFTs, this allows to construct new, exceptional, $\mathcal{N}=3$ theories.

Keywords: Extended Supersymmetry, String Duality, Supersymmetric Gauge Theory, Conformal Field Theory

ARXIV EPRINT: 1611.05769 


\section{Contents}

1 Introduction 1

2 M5 brane construction of known $\mathcal{N}=3$ theories 3

2.1 S-fold construction 3

$2.2 \mathcal{N}=3$ from six dimensions 4

2.2.1 From M5 branes to D3 branes 4

2.2.2 From D3 branes back to M5 branes 5

$\begin{array}{lll}2.2 .3 & \text { Supercharges } & 7\end{array}$

2.3 Field theory interpretation 8

3 New $\mathcal{N}=3$ theories of exceptional type 9

3.1 Six dimensional $(0,2)$ E-type theories from M-theory 9

$\begin{array}{ll}3.2 \text { New } \mathcal{N}=3 \text { theories } & 12\end{array}$

4 Conclusions 13

A Details on the $\mathcal{N}=3$ exceptional quotient $\quad 14$

\section{Introduction}

One of the biggest strengths of string theory lies on its ability to reformulate field theory questions in terms of geometry. In particular cases the relevant geometry is of a particularly tractable form (for instance, a Calabi-Yau manifold), and we can apply powerful techniques in algebraic geometry to study various aspects of the associated field theory. Very well known examples are the Seiberg-Witten solutions of $\mathcal{N}=2 \mathrm{SYM}$ and SQCD with gauge group $\mathrm{SU}(2)[1,2]$, which are beautifully geometrized in string theory in terms of F-theory [3]. More generally, a large number of field theory results can be understood in terms of "geometric engineering" of QFTs [4], which reduces subtle questions in field theory to questions about string theory on specific geometries.

Along somewhat related lines, starting in particular with [5], it has been recently realized that for a particular class of $\mathcal{N}=2$ theories, namely those obtainable from compactification of the six dimensional $(0,2)$ SCFT on a Riemann surface, much of the interesting information of the four dimensional theory can be understood in terms of properties of the compactification space. The resulting formalism is very rich and powerful, and has yielded beautiful insights into the properties of four dimensional field theories. (See [6] for a nice review of some of these developments.)

Nevertheless, it is well known that classical geometry is not the only context in which string theory is well defined, so a natural question to ask is whether the ideas above can be 
extended in an interesting way once we allow ourselves to abandon the realm of classical geometry. A particular case of interest to us here is that of U-manifolds [7-10]: these are spaces which are locally geometric, but which involve string dualities in the transition functions between local patches. Clearly, ordinary geometries are a subclass of such constructions, where the patching functions are diffeomorphisms, but one can reasonably expect the class of non-geometric constructions to be significantly larger than the class of constructions with a geometric interpretation. If this expectation holds, it is then also reasonable to expect that the space of field theories accessible using non-geometric techniques is also significantly larger than that accessible using ordinary geometric constructions.

In this note we aim to give some first steps in this direction, by constructing a class of non-geometric compactifications of M-theory which engineer various interesting field theories. The theories that we construct explicitly in this note are

- The $\mathcal{N}=3$ theories constructed in [11], rewritten as M5 branes on a $T^{2}$ inside a non-geometric compactification.

- The six-dimensional $(0,2)$ SCFTs of exceptional type arising directly from a nongeometric compactification of M-theory down to six dimensions.

- A new class of $\mathcal{N}=3$ theories associated to the exceptional $(0,2)$ theories by compactification on a $T^{2}$ inside a non-geometric background, combining the two constructions above.

The first two classes of theories are already known from geometric constructions, but the third one is new. One can already find evidence for its existence from the four dimensional field theory perspective. Indeed, the construction of the $\mathcal{N}=3$ theories presented in [11], as quotients of $\mathcal{N}=4 \mathrm{U}(N) \mathrm{SYM}$, relies on having an R-symmetry group $\mathrm{SO}(6)_{R}$ together with an enhanced symmetry (for certain values of the coupling) contained in the duality group $\mathrm{SL}(2, \mathbb{Z})$. These properties are not exclusive of the $\mathrm{U}(N)$ theory, but are also present in $\mathcal{N}=4 \mathrm{SYM}$ with gauge group $D_{N}$ or $E_{n}$, which are self-dual under MontonenOlive duality. ${ }^{1,2}$ Thus, it is natural to assume that one can take an $\mathcal{N}=3$ quotient of these $\mathcal{N}=4$ theories. In this note we will focus on the exceptional cases, the generalization to the orthogonal case being straightforward.

We will provide an M-theory construction of these theories in terms of singular Ufolds, which we expect to be intrinsically non-geometric. What we mean by "intrinsically non-geometric" is simply that there is no duality frame in which the system is described

\footnotetext{
${ }^{1}$ The action of duality for non-simply laced groups is more complicated, and in particular it also acts on the moduli space for the $G_{2}$ and $F_{4}$ theories [12]. It would be rather interesting to extend the formalism in this paper to these cases.

${ }^{2}$ More precisely, in the cases of $E_{6}$ and $E_{7}$ the self-dual forms of the group that will appear in our geometric construction are $\left(E_{6} \times \mathrm{U}(1)\right) / \mathbb{Z}_{3}$ and $\left(E_{7} \times \mathrm{U}(1)\right) / \mathbb{Z}_{2}$, with the extra $\mathrm{U}(1)$ factors associated to center-of-mass modes of the string configuration. This is in analogy with the fact that a stack of M5 branes on $T^{2}$ gives rise to a gauge group $\mathrm{U}(N)=(\mathrm{SU}(N) \times \mathrm{U}(1)) / \mathbb{Z}_{N}$, and not simply $\mathrm{SU}(N)$. The difference is important since neither $E_{6}$ or $E_{7}$ are invariant under S-duality, but rather map to their adjoint forms $E_{6} / \mathbb{Z}_{3}$ and $E_{7} / \mathbb{Z}_{2}$.
} 
by string theory in a geometric background. ${ }^{3}$ This does not mean that geometry is entirely useless: as we shall see some aspects of the problem can still be fruitfully geometrized using arguments similar to those in [7] and [13-16].

We will start in $\S 2$ by reconstructing the $\mathcal{N}=3$ theories found in [11] in terms of an M5 wrapping a $T^{2}$ in an M-theory U-fold background. In $\S 3.1$ we will construct the $E$ type $(0,2)$ theories in six dimensions in terms of M-theory compactified on a five-manifold (elaborating on an observation in $[8,17]$ ). We then combine both constructions in $\S 3.2$ in order to engineer the theories of interest. We conclude in $\S 4$ with a discussion of the (numerous) directions for further research.

\section{M5 brane construction of known $\mathcal{N}=3$ theories}

In this section we obtain the four dimensional $\mathcal{N}=3$ theories constructed in [11] (see also [18-23]) from the $(0,2) A_{N-1}$ theory in six dimensions. ${ }^{4}$ Along the way we will be naturally lead to consider non-geometric compactifications of M-theory.

\subsection{S-fold construction}

Let us start by briefly reviewing the construction of the four dimensional $\mathcal{N}=3$ theories of [11]. The basic idea is to take certain quotient of four dimensional $\mathcal{N}=4 \mathrm{SYM}$ with gauge group $\mathrm{U}(N)$ by a $\mathbb{Z}_{k}^{\mathcal{N}=3}$ symmetry of the theory, which includes both R-symmetry rotations and $\operatorname{SL}(2, \mathbb{Z})$ duality. In order for the quotient to make sense, the coupling constant of the original theory must be tuned to a particular value that lies at strong coupling, so that part of the duality group becomes an actual symmetry. The quotient then projects out the corresponding marginal deformation together with four of the supercharges [19, 24]. More specifically, the quotient we need to take is $\mathbb{Z}_{k}^{\mathcal{N}=3}=\mathbb{Z}_{k}^{R} \cdot \mathbb{Z}_{k}^{\tau}$ for $k=3,4,6$, where $\mathbb{Z}_{k}^{R}$ is generated by

$$
R_{k}=\left(\begin{array}{ccc}
\hat{R}_{k}^{-1} & 0 & 0 \\
0 & \hat{R}_{k} & 0 \\
0 & 0 & \hat{R}_{k}
\end{array}\right) \in \mathrm{SO}(6)_{R}
$$

with $\hat{R}_{k}$ being a $2 \pi / k$ rotation in two dimensions. The S-duality quotient $\mathbb{Z}_{k}^{\tau}$ is generated, for $k=3,4,6$, by the following matrices in $\mathrm{SL}(2, \mathbb{Z})$

$$
S_{3}=\left(\begin{array}{ll}
0 & -1 \\
1 & -1
\end{array}\right), \quad S_{4}=\left(\begin{array}{cc}
0 & -1 \\
1 & 0
\end{array}\right), \quad S_{6}=\left(\begin{array}{cc}
1 & -1 \\
1 & 0
\end{array}\right) .
$$

\footnotetext{
${ }^{3}$ Our expectation is based on the fact that the two ingredients we combine for constructing these two theories are geometric in distinct duality frames, or more concretely because we take an $O(5,5 ; \mathbb{Z})$ U-duality action which cannot be conjugated into a geometric subgroup. This shows that our construction cannot be conjugated into pure geometry, but it does not show that a disconnected class of geometric constructions for these exceptional $\mathcal{N}=3$ theories cannot exist.

${ }^{4}$ Strictly speaking, the torus compactification of the $(2,0) A_{N-1}$ theory (as engineered by putting IIB string theory on $\mathbb{C}^{2} / \mathbb{Z}_{N}$, for example) yields a $\mathcal{N}=4 \mathrm{SYM}$ theory with algebra $\mathfrak{s u}(N)$, and thus a gauge group such as $\mathrm{SU}(N)$ or $\mathrm{SU}(N) / \mathbb{Z}_{N}$. In the rest of the paper, when we talk about the $(2,0) A_{N-1}$ theory, we actually mean the six dimensional $(2,0)$ theory living on a stack of M5 branes, whose torus compactification yields $\mathcal{N}=4 \mathrm{U}(N)$ SYM. We will never make use of the "genuine" $A_{N-1}$ theory, so hopefully no confusion will arise from our imprecise use of language.
} 
A simple string construction of these theories is to consider M-theory with $N$ M2 branes in $\underline{\mathbb{R}^{1,2}} \times \mathbb{C}^{3} \times T^{2}$, where the underline denotes the dimensions spanned by the M2 branes. Upon taking the F-theory limit, the M2 branes lift to D3 branes in $\underline{\mathbb{R}^{1,3}} \times \mathbb{C}^{3}$, which realizes four dimensional $\mathcal{N}=4 \mathrm{SYM}$ with gauge group $\mathrm{U}(N)$ in their worldvolume. The key point is that in the M-theory description, both the $\mathrm{SO}(6)_{R}$ R-symmetry group and the $\mathrm{SL}(2, \mathbb{Z})$ duality are manifest geometrically. Thus, one can take a conventional orbifold in the M-theory side, namely $\underline{\mathbb{R}^{1,2}} \times\left(\mathbb{C}^{3} \times T^{2}\right) / \mathbb{Z}_{k}$, which after taking the F-theory limit reproduces the quotient in the four-dimensional gauge theory. This yields an $\mathcal{N}=3$ theory on the worldvolume of a stack of D3 branes probing a generalized orientifold, dubbed S-fold in [22]. ${ }^{5}$

\section{$2.2 \mathcal{N}=3$ from six dimensions}

Now we would like to obtain the four dimensional $\mathcal{N}=3$ theories from the $(0,2) A_{N-1}$ superconformal field theory in six dimensions. The R-symmetry group of these theories is $\mathrm{SO}(5)_{R}$ and the supercharges transform in the $(4,4)$ of $\mathrm{SO}(5,1) \times \mathrm{SO}(5)_{R}$.

\subsubsection{From M5 branes to D3 branes}

Four dimensional $\mathcal{N}=4 \mathrm{SYM}$ with gauge group $\mathrm{U}(N)$ can be obtained from the $(0,2)$ $A_{N-1}$ theory by compactifying it on a torus [25]. In this construction, the S-duality group $\mathrm{SL}(2, \mathbb{Z})$ of the four dimensional theory is manifest geometrically, as the group of large diffeomorphisms acting on the torus. In contrast, the full R-symmetry group $\mathrm{SO}(6)_{R}$ is only present in the limit in which the size of the torus vanishes, where $\mathrm{SO}(5)_{R}$ enhances to $\mathrm{SO}(6)_{R}$. Since the quotient we want to take in the $\mathcal{N}=4$ theory involves a subgroup of $\mathrm{SO}(6)_{R}$ which is not in $\mathrm{SO}(5)_{R}$, it is not immediately clear how to proceed in terms of the $(0,2)$ theory when the size of the torus is finite. In order to do so, we need to make the symmetry we want to quotient by manifest in the UV. In the following we do so by starting with a specific M-theory configuration of M5 branes and interpret the result in field theory terms afterwards.

Let us consider a system of $N$ M5 branes on $\mathbb{R}^{1,3} \times S_{M}^{1} \times S_{T}^{1} \times S_{E}^{1} \times \mathbb{C}^{2}$. If we reduce along the M-theory circle $S_{M}^{1}$, we have Type IIA with $N$ D4 branes on $\mathbb{R}^{1,3} \times S_{T}^{1} \times S_{E}^{1} \times \mathbb{C}^{2}$.

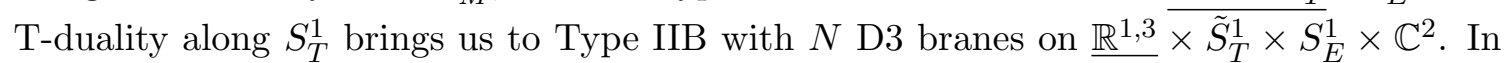
this last picture we see that, due to the two circles in the transverse space to the D3 branes, the R-symmetry group $\mathrm{SO}(6)_{R}$ is broken, and is only recovered in the IR where these circles decompactify, as mentioned earlier. In general, we expect it to be broken to $\mathrm{SO}(4) \times \mathbb{Z}_{2}$, where $\mathrm{SO}(4)$ acts on $\mathbb{C}^{2}$ and $\mathbb{Z}_{2}$ acts by reflection on the torus $\tilde{T}_{E}^{2}=\tilde{S}_{T}^{1} \times S_{E}^{1}$. However, if we tune the complex structure $\tilde{\tau}_{E}$ of $\tilde{T}_{E}^{2}$ to be, for example, $\tilde{\tau}_{E}=i$, we see that the symmetry group is enhanced to $\mathrm{SO}(4) \times \mathbb{Z}_{4}$, with $\mathbb{Z}_{4}$ acting on $\tilde{T}_{E}^{2}$ as a rotation by $\pi / 2$, namely

$$
\mathbb{Z}_{4}:\left(\tilde{x}_{T}, x_{E}\right) \longrightarrow\left(-x_{E}, \tilde{x}_{T}\right)
$$

\footnotetext{
${ }^{5}$ We restrict to the case without torsion fluxes in the whole paper. A systematic discussion of fluxes in S-folds can be found in [22]. As we discuss in the conclusions, we expect that some or all of these discrete degrees of freedom can be encoded into a discrete twist along the compactification torus.
} 
where $\left(\tilde{x}_{T}, x_{E}\right)$ are coordinates on $\tilde{T}_{E}^{2}$. Thus, in that particular situation, even though we do not have the full R-symmetry $\mathrm{SO}(6)_{R}$, we have precisely the $\mathbb{Z}_{4}^{R}$ within $\mathrm{SO}(6)_{R}$ that we need in the construction of the $\mathcal{N}=3$ theories. Similarly, if we tune $\tilde{\tau}_{E}=e^{i \pi / 3}$, we have an enhanced $\mathbb{Z}_{6}$ R-symmetry, which allows to construct the rest of the $\mathcal{N}=3$ theories in [11]. In the following we restrict to the case $\mathbb{Z}_{4}$ for simplicity, with generalization to the other cases being straightforward.

Since the original description in terms of M5 branes is dual to the one involving D3 branes, we know that such $\mathbb{Z}_{4}$ symmetry must be there too. In order to identify it in terms of M5 branes, we dualize back tracing carefully the $\mathbb{Z}_{4}$ that acts on the torus $\tilde{T}_{E}^{2}$.

\subsubsection{From D3 branes back to M5 branes}

The first step is to T-dualize along $\tilde{S}_{T}^{1}$, which gives Type IIA with $N$ D4 branes on $\frac{\mathbb{R}^{1,3} \times S_{T}^{1}}{\mathrm{Ka}} \times S_{E}^{1} \times \mathbb{C}^{2}$. Since upon T-duality we exchange $\tau \leftrightarrow \rho$, the complexified Kähler parameter

$$
\rho=\int_{T_{E}^{2}} B+i \sqrt{\operatorname{det} G}
$$

of $T_{E}^{2}=S_{T}^{1} \times S_{E}^{1}$ is equal to $\rho_{E}=i$. Here $G$ is the metric on the torus $T_{E}^{2}$. In particular, this means that the radii of $T_{E}^{2}$ (in string frame) are constrained by $r_{T} r_{E}=1$ and that the NSNS B-field is zero when integrated over $T_{E}^{2}$.

In this picture, the $\mathbb{Z}_{4}$ symmetry (2.3) is no longer geometric since, for the closed string sector, it exchanges momentum states in one direction with winding modes in the other. Type IIA on a two-torus has a T-duality group $O(2,2 ; \mathbb{Z})$, which can be written as

$$
O(2,2 ; \mathbb{Z})=\left(\mathrm{SL}(2, \mathbb{Z})_{\tau_{E}} \times \mathrm{SL}(2, \mathbb{Z})_{\rho_{E}}\right) \rtimes\left(\mathbb{Z}_{2}^{\tau_{E} \leftrightarrow \rho_{E}} \times \mathbb{Z}_{2}^{\left(\tau_{E}, \rho_{E}\right) \leftrightarrow\left(-\bar{\tau}_{E},-\bar{\rho}_{E}\right)}\right) .
$$

Here $\operatorname{SL}(2, \mathbb{Z})_{\tau_{E}}$ acts geometrically on $T_{E}^{2}$, while $\operatorname{SL}(2, \mathbb{Z})_{\rho_{E}}$ does it non-geometrically, since it acts on $\rho_{E}$, which contains the volume modulus, by the usual linear fractional transformations. Generically, this is a duality and not a symmetry, in the sense that it identifies states with different values of the fields at infinity. However, for the special value $\rho_{E}=i$, we see that the $\mathbb{Z}_{4} \subset \mathrm{SL}(2, \mathbb{Z})_{\rho}$, acting as ${ }^{6}$

$$
\rho_{E} \longrightarrow-\frac{1}{\rho_{E}},
$$

becomes a symmetry, since it leaves the asymptotic value of all the fields fixed. Thus, for $\rho_{E}=i$, we may take the quotient by $\mathbb{Z}_{4}$. Regarding the action on the open string degrees of freedom of the D4 branes, at the massless level, this $\mathbb{Z}_{4}$ exchanges the position along the transverse circle $S_{E}^{1}$ with the Wilson line along $S_{T}^{1}$.

The next step is to take the M-theory lift of this configuration and, in particular, of the $\mathbb{Z}_{4}$ action (2.6). As explained in [26], the duality group of M-theory on $T^{3}$ is given by $\mathrm{SL}(3, \mathbb{Z}) \times \mathrm{SL}(2, \mathbb{Z})_{\rho}$, where the first factor acts in the natural way on $T^{3}$ and the second

\footnotetext{
${ }^{6} \mathrm{~A}$ useful way of viewing this transformation is as a T-duality along $S_{T}^{1}$, followed by a T-duality along $S_{E}^{1}$, followed by a rotation exchanging the two coordinates.
} 
corresponds to certain electric-magnetic duality in eight dimensions. Indeed, it exchanges the M-theory three-form $C$ along the eight non-compact dimensions with its Hodge-dual potential or, equivalently, maps unwrapped M2 branes to wrapped M5 branes. In addition, it acts on the M-theory $\rho$ parameter by linear fractional transformations, where $\rho$ is given now by

$$
\rho=\int_{T^{3}} C+i \sqrt{\operatorname{det} G}
$$

with $G$ the metric on $T^{3}$. This provides the M-theory lift of the T-duality group of Type IIA on $T^{2}$.

Thus, we find that the M-theory lift consists of $N$ M5 branes on $\mathbb{R}^{1,3} \times S_{M}^{1} \times S_{T}^{1} \times$ $S_{E}^{1} \times \mathbb{C}^{2}$ with $\rho=i$, where $\rho$ is now the M-theory modulus. Having $\rho=\overline{i \text { implies that }}$

$$
R_{M} R_{T} R_{E}=1
$$

where $R_{M}, R_{T}, R_{E}$ are the radii of $S_{M}^{1}, S_{T}^{1}, S_{E}^{1}$ in the M-theory metric.

As we mentioned earlier, the S-duality group $\operatorname{SL}(2, \mathbb{Z})$ of four dimensional $\mathcal{N}=4 \mathrm{SYM}$ arises from large diffeomorphisms of the torus wrapped by the M5 branes. Thus, in order to have $\mathbb{Z}_{4}^{\tau}$ within $\mathrm{SL}(2, \mathbb{Z})$ to be an actual symmetry, we have to tune the complex structure $\tau$ of $S_{M}^{1} \times S_{T}^{1}$ to be $\tau=i$ which, in particular, implies that

$$
R_{M}=R_{T} .
$$

Thus, requiring the presence of both the R-symmetry $\mathbb{Z}_{4}^{R}$ (eq.(2.8)) and the S-duality $\mathbb{Z}_{4}^{\tau}$ (eq.(2.9)) leaves only one free parameter, namely

$$
R_{M}=R, \quad R_{T}=R, \quad R_{E}=\frac{1}{R^{2}} .
$$

Notice that the $\mathbb{Z}_{4}^{\mathcal{N}=3}$ action we want to quotient by in four dimensions to construct the $\mathcal{N}=3$ theories is present for every value of $R$. The four dimensional superconformal theory is obtained when $R \rightarrow 0$. In this limit, the two-torus wrapped by the M5 branes becomes small while the transverse circle decompactifies.

To summarize, we can obtain the four dimensional $\mathcal{N}=3$ theories in this context by considering $N$ M5 branes probing certain non-geometric singularity. More explicitly, we need to consider $N$ M5 branes on $\mathbb{R}^{1,3} \times\left(S_{M}^{1} \times S_{T}^{1} \times S_{E}^{1} \times \mathbb{C}^{2}\right) / \mathbb{Z}_{k}$, where $\mathbb{Z}_{k}$ is the combined action

$$
\mathbb{Z}_{k}=\mathbb{Z}_{k}^{R} \cdot \tilde{\mathbb{Z}}_{k}^{R} \cdot \mathbb{Z}_{k}^{\tau}
$$

Here $\mathbb{Z}_{k}^{R}$ is a rotation acting on $\mathbb{C}^{2}$ generated by

$$
R_{k}=\left(\begin{array}{cc}
\hat{R}_{k}^{-1} & 0 \\
0 & \hat{R}_{k}
\end{array}\right),
$$

where $\hat{R}_{k}$ is a $2 \pi / k$ rotation in two dimensions. Moreover, $\tilde{\mathbb{Z}}_{k}^{R}$ is a non-geometric quotient generated by acting on the $\rho$ parameter of $T^{3}=S_{M}^{1} \times S_{T}^{1} \times S_{E}^{1}$, which fixes $\rho$ to a specific value such that the volume of $T^{3}$ is of order one. Finally, $\mathbb{Z}_{k}^{\tau}$ acts on $T^{2}=S_{M}^{1} \times S_{T}^{1}$ as $u \rightarrow e^{2 \pi i / k} u$, where $u$ is a flat complex coordinate on $T^{2}$. This fixes the complex structure of the torus to a particular value. 


\subsubsection{Supercharges}

It is interesting to compute the amount of supersymmetry preserved by the configuration described above directly. If we consider $N$ M5 branes on $\mathbb{R}^{1,3} \times S_{M}^{1} \times S_{T}^{1} \times S_{E}^{1} \times \mathbb{C}^{2}$, the sixteen supercharges preserved by the M5 branes transform as $[7]^{7}$

$$
\left(S_{4}^{+}, S_{4}^{+}\right)_{-\frac{1}{2}, \frac{1}{2}} \oplus\left(S_{4}^{+}, S_{4}^{-}\right)_{-\frac{1}{2},-\frac{1}{2}} \oplus\left(S_{4}^{-}, S_{4}^{+}\right)_{\frac{1}{2},-\frac{1}{2}} \oplus\left(S_{4}^{-}, S_{4}^{-}\right)_{\frac{1}{2}, \frac{1}{2}}
$$

under

$$
\mathrm{SO}(1,3) \times \mathrm{SO}(4)_{R} \times \mathrm{U}(1)_{\tau} \times \mathrm{U}(1)_{\rho}
$$

where $S_{4}^{ \pm}$are the positive/negative chirality spinors of either $\mathrm{SO}(1,3)$ or $\mathrm{SO}(4)$. Furthermore, $\mathrm{U}(1)_{\tau}$ corresponds to the rotations on the torus wrapped by the M5 branes and $\mathrm{U}(1)_{\rho}$ is the bundle associated to the duality group $\mathrm{SL}(2, \mathbb{Z})_{\rho}$, defined as follows. Given an $\mathrm{SL}(2, \mathbb{Z})_{\rho}$ bundle with transition functions

$$
M=\left(\begin{array}{ll}
a & b \\
c & d
\end{array}\right) \in \mathrm{SL}(2, \mathbb{Z})_{\rho}
$$

the $\mathrm{U}(1)_{\rho}$ bundle is obtained by using transition functions $e^{i \arg (c \rho+d)}$.

We can compute how the supercharges transform under the discrete group (2.11) and those that are not invariant will be projected out. More explicitly, $\mathbb{Z}_{k}^{R}$ acts on the supercharges as

$$
\begin{aligned}
\left(S_{4}^{ \pm}, S_{4}^{+}\right)_{p, q} & \rightarrow \quad\left(S_{4}^{ \pm}, S_{4}^{+}\right)_{p, q} \\
\mathbb{Z}_{k}^{R}:\left(S_{4}^{ \pm},\left(+\frac{1}{2},-\frac{1}{2}\right)\right)_{p, q} & \rightarrow e^{-2 \pi i / k}\left(S_{4}^{ \pm},\left(+\frac{1}{2},-\frac{1}{2}\right)\right)_{p, q} \\
\left(S_{4}^{ \pm},\left(-\frac{1}{2},+\frac{1}{2}\right)\right)_{p, q} & \rightarrow e^{2 \pi i / k}\left(S_{4}^{ \pm},\left(-\frac{1}{2},+\frac{1}{2}\right)\right)_{p, q} .
\end{aligned}
$$

Under the non-geometric action $\tilde{\mathbb{Z}}_{k}^{R}$ we find

$$
\tilde{\mathbb{Z}}_{k}^{R}: \begin{aligned}
& \left(S_{4}^{ \pm}, \bullet\right)_{p, \frac{1}{2}} \rightarrow e^{\pi i / k}\left(S_{4}^{ \pm}, \bullet\right)_{p, \frac{1}{2}} \\
& \left(S_{4}^{ \pm}, \bullet\right)_{p,-\frac{1}{2}} \rightarrow e^{-\pi i / k}\left(S_{4}^{ \pm}, \bullet\right)_{p,-\frac{1}{2}}
\end{aligned}
$$

where the bullets stand for omitted $S_{4}^{ \pm}$terms. Finally, under the rotation of the torus wrapped by the M5 branes we find that

$$
\mathbb{Z}_{k}^{\tau}: \begin{gathered}
\left(S_{4}^{ \pm}, \bullet\right)_{\frac{1}{2}, q} \rightarrow e^{\pi i / k}\left(S_{4}^{ \pm}, \bullet\right)_{\frac{1}{2}, q} \\
\left(S_{4}^{ \pm}, \bullet\right)_{-\frac{1}{2}, q} \rightarrow e^{-\pi i / k}\left(S_{4}^{ \pm}, \bullet\right)_{-\frac{1}{2}, q}
\end{gathered}
$$

Under the combined action $\mathbb{Z}_{k}=\mathbb{Z}_{k}^{R} \cdot \tilde{\mathbb{Z}}_{k}^{R} \cdot \mathbb{Z}_{k}^{\tau}$, only twelve supercharges remain invariant so we have $\mathcal{N}=3$, as expected.

\footnotetext{
${ }^{7}$ In our conventions, the M5 brane preserves $(0,2)$ supersymmetry in six dimensions.
} 


\subsection{Field theory interpretation}

Up to now we have discussed how to obtain the four dimensional $\mathcal{N}=3$ from six dimensions by using the M-theory construction in terms of M5 branes. However, it should be possible to understand this procedure directly in terms of the $(0,2)$ theory. For concreteness we will discuss the case $k=4$ in the following but the other cases work analogously.

Let us start by looking at the moduli space of the abelian $(0,2)$ theory (just one M5 brane in flat space), which is

$$
\mathcal{M}=\mathbb{R}^{5}
$$

The R-symmetry group $\mathrm{SO}(5)_{R}$ acts on the moduli space in the obvious way. Notice that there is an additional $\mathbb{Z}_{2}$ symmetry of the theory that acts as the element $(-1) \in O(5)$ on the moduli space and which, in order commute with the supercharges, must act also with a minus sign on the self-dual two-form potential $B$. In the M-theory construction, this corresponds to the possibility of taking an M5 brane on an orbifold $\mathbb{R}^{5} / \mathbb{Z}_{2}$. Since the resulting space is non-orientable, the orbifold action must be accompanied by $C \rightarrow-C$ [27], which induces $B \rightarrow-B$ on the M5 brane. Gauging such $\mathbb{Z}_{2}$ provides the field theory construction of the $(0,2) D$-type theories in terms of the $A$-type ones. ${ }^{8}$ Thus, the full symmetry group of the theory is $\mathrm{SO}(5)_{R} \times \mathbb{Z}_{2}$, the same as the isometry group of the moduli space that leaves the origin fixed.

When we compactify the theory on a square torus $T^{2}=S_{M}^{1} \times S_{T}^{1}$, the moduli space becomes

$$
\mathcal{M}=\mathbb{R}^{5} \times S_{h}^{1},
$$

where $S_{h}^{1}$ corresponds to the scalar $\varphi$ that comes from the holonomy of the two-form potential along $T^{2}$, namely

$$
\exp \left[i \int_{T_{\tau}^{2}} B\right]=\exp \left[i R_{M} R_{T} \varphi\right],
$$

where $R_{M}$ and $R_{T}$ are the radii of the torus. From this we see that the radius of the circle $S_{h}^{1}$ in moduli space is $\left(R_{M} R_{T}\right)^{-1}$ and that this corresponds to turning on a relevant deformation in the six dimensional theory. If we compactify a direction transverse to the M5 $\left(\mathbb{R} \rightarrow S_{p}^{1}\right)$, the moduli space is

$$
\mathcal{M}=\mathbb{R}^{4} \times S_{p}^{1} \times S_{h}^{1}
$$

with $S_{p}^{1}$ of radius $R_{E}$. This amounts to turning on an irrelevant deformation of the $(0,2)$ theory that breaks the R-symmetry group from $\mathrm{SO}(5)_{R}$ to $\mathrm{SO}(4)_{R}$, so the full symmetry group is generically $\mathrm{SO}(4)_{R} \times \mathbb{Z}_{2}$. Since the $\mathbb{Z}_{2}$ acts on the potential as $B \rightarrow-B$, it acts by reversing all the coordinates in the moduli space (2.22).

\footnotetext{
${ }^{8}$ Strictly speaking, this provides a variant of the $D$-type theory in which the global $\mathbb{Z}_{2}$ symmetry of the $D_{N}$ theory is gauged, analogous to the difference between a gauge theory with gauge group $\mathrm{SO}(2 N)$ and $O(2 N)$. This subtlety becomes more clear when one looks carefully at the different possible boundary conditions of the holographic dual [22].
} 
From the M-theory construction discussed above, we expect that for a particular value of this irrelevant deformation $\left(R_{E}\right)$, there is an enhancement of the symmetry group of the theory to $\mathrm{SO}(4)_{R} \times \mathbb{Z}_{4}$. One can find evidence for this directly in field theory by looking at the isometries of moduli space (2.22). Indeed, the two $S^{1}$ factors form a complex torus $T_{p h}^{2}=S_{p}^{1} \times S_{h}^{1}$ with complex structure $\tau_{p h}$. When $\tau_{p h}=i$, there is an enhancement of the isometry group of the moduli space to $\mathrm{SO}(4)_{R} \times \mathbb{Z}_{4}$. In particular, this happens when the radii of $S_{p}^{1}$ and $S_{h}^{1}$ are the same, namely when $R_{E}=\left(R_{M} R_{T}\right)^{-1}$, cf. (2.8). Thus, we conclude that the complex structure $\tau_{p h}$ is precisely the same as the $\rho$ parameter of the M-theory construction presented above. The enhanced discrete symmetry acts as

$$
\mathbb{Z}_{4}:(\phi, \varphi) \rightarrow(-\varphi, \phi)
$$

where $\phi$ is the position along the transverse circle. We see that it exchanges the position modulus along $S_{E}^{1}$ with the holonomy coming from $B$ on $S_{M}^{1} \times S_{T}^{1}$, as expected.

In the simplest construction of the class $\mathcal{S}$ theories [5], the transverse space to the M5 branes is taken to be $\mathbb{R}^{5}$. This means that the full R-symmetry group $\mathrm{SO}(5)_{R}$ can be used to topologically twist the theory and preserve supersymmetry. In our case, by compactifying one of the directions in the transverse space, we break the R-symmetry to $\mathrm{SO}(4)_{R}$, but for special values of the transverse radius, there is an enhancement to $\mathrm{SO}(4)_{R} \times \mathbb{Z}_{4}$, which is not contained in $\mathrm{SO}(5)_{R}$. This extra symmetry can be used to perform a compactification such that the four dimensional theory preserves $\mathcal{N}=3$ supersymmetry. In this sense, our construction can be regarded as a generalization of the class $\mathcal{S}$ theories.

\section{New $\mathcal{N}=3$ theories of exceptional type}

\subsection{Six dimensional (0,2) E-type theories from M-theory}

The six dimensional $(0,2)$ ADE theories first appeared as the low energy description of ADE singularities in Type IIB [25]. Such singularities are locally of the form $\mathbb{C}^{2} / \Gamma$, where $\Gamma$ is a finite subgroup of $\mathrm{SU}(2)$. However, in order to provide an M-theory realization of these theories, it is useful to consider instead a singular elliptic fibration over $\mathbb{C}$ such that, upon decompactification of the fiber, we recover $\mathbb{C}^{2} / \Gamma{ }^{9}$ As we go around the singularity, the fiber undergoes a monodromy given by an element of $\operatorname{SL}(2, \mathbb{Z})_{\tau}$, which characterizes uniquely the type of singularity of the corresponding Weierstrass model (see table 1). In particular, such a monodromy acts on the complex structure of the fiber as

$$
\tau \rightarrow \frac{a \tau+b}{c \tau+d}, \quad\left(\begin{array}{ll}
a & b \\
c & d
\end{array}\right) \in \operatorname{SL}(2, \mathbb{Z})_{\tau} .
$$

Away from the singular point, the space is locally given by $\mathbb{C} \times T^{2}$ and the group $\mathrm{SL}(2, \mathbb{Z})_{\tau}$ is part of the T-duality group of Type IIB on $T^{2}$, see eq.(2.5).

\footnotetext{
${ }^{9}$ In our construction the transverse geometry has some compact directions, so the four dimensional theory arising upon compactification of the $6 \mathrm{~d} A_{N-1}$ theory includes a free $\mathrm{U}(1)$ multiplet, i.e. we have the $\mathrm{U}(N)$ theory.
} 


\begin{tabular}{|c|c|c|c|c|c|}
\hline & $A_{N-1}$ & $\overline{D_{N}}$ & $E_{6}$ & $E_{7}$ & $E_{8}$ \\
\hline \multirow{2}{*}{ Monodromy } & $(1 N)$ & $(-14-N)$ & $0-1$ & $0-1$ & $(1-1)$ \\
\hline & $\left(\begin{array}{ll}0 & 1\end{array}\right)$ & -1 & $(1-1)$ & 10 & $\left(\begin{array}{ll}1 & 0\end{array}\right)$ \\
\hline
\end{tabular}

Table 1. Monodromy of ADE singularities in elliptic fibrations. For the case $D_{N}$ we restrict $N \geq 4$.

If we T-dualize along one of the directions of the fiber, we map Type IIB to Type IIA and exchange $\tau \leftrightarrow \rho$, which means that we have an elliptic fibration with a monodromy acting on $\rho$, not on $\tau$. This is an example of a non-geometric space, sometimes referred to as a T-fold.

As shown in table 1, the corresponding monodromy for the case of $A_{N-1}$ acts on the Kähler parameter as $\rho \rightarrow \rho+N$, i.e. a shift in the $B$-field. This means that the singularity is magnetically charged under $B$ which, together with having sixteen preserved supercharges, implies that such an object is a stack of NS5 branes. Notice that since the monodromy does not act on the volume of $T^{2}$, we may decompactify it.

For the case of $D_{N}$, the action on $\rho$ is the same as for $A_{N-3}$, but the monodromy differs by an overall sign. This corresponds to having $N$ NS5 branes in the presence of an ON5, defined as Type IIA modded out by $I_{4}(-1)^{F_{L}}[28]$.

For the exceptional cases, the interpretation is rather different. Let us consider for concreteness the case of $E_{7}$, for which the monodromy acts as $\rho \rightarrow-1 / \rho$. Unlike the previous cases, this involves a genuine stringy duality that (for vanishing $B$-field) sends the volume of the fiber to its inverse. In particular, this implies that, at the singularity, the value of $\rho$ is given by the fixed point of $\rho \rightarrow-1 / \rho$, namely $\rho=i$. Thus, we cannot decompactify the fiber, in contrast with the $A_{N}$ and $D_{N}$ cases. For $E_{6}$ and $E_{8}$, the $\rho$ modulus is fixed at the singularity to $\rho=e^{i \pi / 3}$.

This shows that all the six dimensional $(0,2)$ theories can be engineered in Type IIA, as long as we allow to have singular non-geometric compactification spaces.

Now that we have a Type IIA construction of these theories, we can obtain the M-theory lift, as done in the previous section. The non-geometric action on the torus $T^{2}$ in Type IIA lifts to a non-geometric action on the M-theory three torus $T^{3}[26]$. Thus, we find that we can engineer the $(0,2)$ ADE theories in M-theory by considering a nongeometric $T^{3}$ fibration over $\mathbb{C}$, where the monodromy acts on the complexified volume of $T^{3}$, as in table 1 .

Similarly to the Type IIA case, we can interpret the $A_{N-1}$ and $D_{N}$ cases as corresponding to a stack of $N$ M5 branes, either in flat space or in an orbifold $\mathbb{R}^{5} / \mathbb{Z}_{2}$ [28]. However, for the exceptional cases the monodromy acts non-trivially on the volume of $T^{3}$, so we cannot decompactify the fiber. In these cases, the six dimensional superconformal point is reached, in the IIB description, when we decompactify the fiber, namely $\rho_{\text {IIB }} \rightarrow i \infty$, keeping $g_{\mathrm{IIB}}$ fixed. In terms of the M-theory data, this corresponds (for $E_{7}$ ) to taking the limit $R \rightarrow 0$ in

$$
R_{A}=R^{-2} c^{-1}, \quad R_{T}=R c^{-1}, \quad R_{M}=R c^{2},
$$


where $c=\left(g_{I I B}\right)^{\frac{1}{3}}$ and $R_{A}, R_{T}, R_{M}$ are the radii of $T^{3}=S_{A}^{1} \times S_{T}^{1} \times S_{M}^{1}$ in the M-theory metric. Here $S_{T}^{1}$ is the circle along which we T-dualize and $S_{M}^{1}$ is the M-theory circle. Notice that the M-theory complexified volume is $\rho=i$ for every $c$ and $R$ in (3.2).

Since the M-theory configuration is dual to the original Type IIB setup, we know that it preserves sixteen supercharges of the same chirality. However, it is instructive to compute this directly in M-theory for the exceptional cases. Consider for the moment M-theory on $\mathbb{R}^{1,5} \times\left(\mathbb{C} \times T^{3}\right)$, so the supercharges transform as

$$
\left(S_{6}^{+}, \frac{1}{2}, \frac{1}{2}, \mathbf{2}\right) \oplus\left(S_{6}^{+},-\frac{1}{2},-\frac{1}{2}, \mathbf{2}\right) \oplus\left(S_{6}^{-}, \frac{1}{2},-\frac{1}{2}, \mathbf{2}\right) \oplus\left(S_{6}^{-},-\frac{1}{2}, \frac{1}{2}, \mathbf{2}\right)
$$

under $\mathrm{SO}(1,5) \times \mathrm{U}(1)_{\mathbb{C}} \times \mathrm{U}(1)_{\rho} \times \mathrm{SU}(2)$, where $\mathrm{U}(1)_{\mathbb{C}}$ is the rotation group in $\mathbb{C}$ and $S_{6}^{ \pm}$are positive/negative chirality spinors in six dimensions. Here $\mathrm{U}(1)_{\rho} \times \mathrm{SU}(2)$ is the maximal compact subgroup of the (continuous version of the) duality group $\mathrm{SL}(2, \mathbb{Z})_{\rho} \times \mathrm{SL}(3, \mathbb{Z})$.

In order to compute the supercharges that survive the quotient, we may regard the non-geometric $T^{3}$ fibration over $\mathbb{C}$ as $\left(\mathbb{C} \times T^{3}\right) / \mathbb{Z}_{p}$ where $\mathbb{Z}_{p}$ acts non-geometrically. Namely, it is given by the combined action

$$
\mathbb{Z}_{p}=\mathbb{Z}_{p}^{\mathbb{C}} \cdot \mathbb{Z}_{p}^{\rho},
$$

where $\mathbb{Z}_{p}^{\mathbb{C}}$ acts on $\mathbb{C}$ as a rotation by $2 \pi / p$ and $\mathbb{Z}_{p}^{\rho}$ acts on $\rho$ via the monodromy in table 1 . We have that $p=3,4,6$ correspond to $E_{6}, E_{7}$ and $E_{8}$, respectively. This is analogous to the statement that the original Weierstrass model for the singular elliptic fibrations of type $I V^{*}, I I I^{*}$ and $I I^{*}$ is birational to orbifolds of the form $\left(\mathbb{C} \times T^{2}\right) / \mathbb{Z}_{p}$, for $p=3,4,6$. In the geometric case, the Weierstrass model and the orbifold are not physically equivalent, since the singularity structure is different in each case, and thus the corresponding superconformal field theories are also different. However, the amount of supersymmetry preserved is indeed the same in both cases so in the following, for simplicity, we will use an orbifold description of the U-fold, which correctly encodes the monodromies associated to the singularity, to compute the amount of supersymmetry preserved. As we will see, this gives the expected number of supercharges. We stress, however, that this is not the U-fold that yields the E-type superconformal field theories we are interested in, which is rather given by the U-dual of the Weierstrass model which one needs for constructing the exceptional $(0,2)$ theories.

On the one hand, under the rotation in $\mathbb{C}$, the supercharges (3.3) transform as

$$
\mathbb{Z}_{p}^{\mathbb{C}}: \begin{aligned}
& \left(S_{6}^{+}, \pm \frac{1}{2}, \pm \frac{1}{2}, \mathbf{2}\right) \rightarrow e^{ \pm i \pi / p}\left(S_{6}^{+}, \pm \frac{1}{2}, \pm \frac{1}{2}, \mathbf{2}\right) \\
& \left(S_{6}^{-}, \pm \frac{1}{2}, \mp \frac{1}{2}, \mathbf{2}\right) \rightarrow e^{ \pm i \pi / p}\left(S_{6}^{-}, \pm \frac{1}{2}, \mp \frac{1}{2}, \mathbf{2}\right) .
\end{aligned}
$$

On the other hand, under the non-geometric monodromy in the fiber, these transform as

$$
\mathbb{Z}_{p}^{\rho}: \begin{aligned}
& \left(S_{6}^{+}, \pm \frac{1}{2}, \pm \frac{1}{2}, \mathbf{2}\right) \rightarrow e^{ \pm i \pi / p}\left(S_{6}^{+}, \pm \frac{1}{2}, \pm \frac{1}{2}, \mathbf{2}\right) \\
& \left(S_{6}^{-}, \pm \frac{1}{2}, \mp \frac{1}{2}, \mathbf{2}\right) \rightarrow e^{\mp i \pi / p}\left(S_{6}^{-}, \pm \frac{1}{2}, \mp \frac{1}{2}, \mathbf{2}\right) .
\end{aligned}
$$

Clearly, the combined action (3.4) preserves all the supercharges of negative chirality and projects out the rest, which gives $(0,2)$ supersymmetry, as expected. 


\subsection{New $\mathcal{N}=3$ theories}

As we mentioned in the introduction, the idea of quotienting $\mathcal{N}=4$ SYM by a combination of appropriate $R$-symmetry and $\mathrm{SL}(2, \mathbb{Z})$ transformations does not require the original group to be $\mathrm{U}(N)$, and should extend in particular to $\mathcal{N}=4$ theories with exceptional gauge groups, which are also self-dual for certain values of the coupling (with the subtlety mentioned in footnote 1 taken into account). This field theory argument suggests that the quotient exists and yields $\mathcal{N}=3$ theories. However, with current technology it is difficult to analyze the resulting theories directly in field theory from the quotient viewpoint, which is one reason why having a string realization is useful.

While the construction presented in [11] using D3 branes is well suited to get the quotient of the $\mathrm{U}(N)$ theory, it does not seem to generalize to the exceptional cases, since there is no known construction of $\mathcal{N}=4$ E-type SYM using D3 branes. However, as we will see in the following, one can obtain a string realization of these by combining appropriately the non-geometric construction of the $(0,2)$ E-type theories presented above with the $\mathcal{N}=3$ quotient of section 2.2. Let us start with M-theory on a five-torus, $\mathbb{R}^{1,3} \times S_{a}^{1} \times S_{b}^{1} \times S_{c}^{1} \times S_{d}^{1} \times S_{e}^{1} \times \mathbb{C}$, where we will denote the different subtori as $T_{a b}^{2}=$ $S_{a}^{1} \times S_{b}^{1}$, etc.

E-type quotient. As explained in the previous section, the non-geometric orbifold that encodes the monodromies associated to the U-fold that yields the E-type $(0,2)$ theories is $\mathbb{R}^{1,3} \times T_{a b}^{2} \times\left(T_{c d e}^{3} \times \mathbb{C}\right) / \mathbb{Z}_{p}^{E}$, where $\mathbb{Z}_{p}^{E}$ acts as in (3.4), which we repeat here for convenience.

$$
\mathbb{Z}_{p}^{E}=\mathbb{Z}_{p}^{\mathbb{C}} \cdot \mathbb{Z}_{p}^{\rho}
$$

where $\mathbb{Z}_{p}^{\mathbb{C}}$ acts on $\mathbb{C}$ as a rotation by $2 \pi / p$ and $\mathbb{Z}_{p}^{\rho}$ acts on the $\rho$ parameter of $T_{\text {cde }}^{3}$ (which we denote by $\rho_{E}$ ) by the monodromy in table 1 . For $p=3,4,6$ we obtain the $(0,2) E_{6,7,8}$ theory on $\mathbb{R}^{1,3} \times T_{a b}^{2}$. This quotient requires $\rho_{E}$ to be either $\rho_{E}=e^{i \pi / 3}$ for $p=3,6$ or $\rho_{E}=i$ for $p=4$.

S-fold quotient. The non-geometric quotient, discussed in section 2.2, that produces the S-fold is given by $\mathbb{R}^{1,3} \times\left(T_{a b c}^{3} \times T_{d e}^{2} \times \mathbb{C}\right) / \mathbb{Z}_{k}^{S}$, where $\mathbb{Z}_{k}^{S}$ is the combined action

$$
\mathbb{Z}_{k}^{S}=\mathbb{Z}_{k}^{R} \cdot \tilde{\mathbb{Z}}_{k}^{R} \cdot \mathbb{Z}_{k}^{\tau}
$$

Here $\mathbb{Z}_{k}^{R}$ is a rotation by $2 \pi / k$ on $T_{d e}^{2} \times \mathbb{C}$, as in (2.12), $\tilde{\mathbb{Z}}_{k}^{R}$ acts on the $\rho$ parameter of $T_{a b c}^{3}$ (denoted by $\rho_{S}$ ) via the monodromy in table 1 and $\mathbb{Z}_{k}^{\tau}$ is a $2 \pi / k$ rotation on $T_{a b}^{2}$. In order to being able to perform this quotient, we need to set $\rho_{S}=\tau_{a b}=\tau_{d e}=e^{i \pi / 3}$ for $k=3,6$ and $\rho_{S}=\tau_{a b}=\tau_{d e}=i$ for $k=4$.

Thus, we conclude that the four dimensional exceptional $\mathcal{N}=3$ theories arise when we take the (non-geometric) S-fold quotient of the U-fold that produces the E-type $(2,0)$ theories. In four dimensional field theory terms, the label $p$ denotes the parent gauge group $E_{6,7,8}$ and the label $k$ is the kind of $\mathcal{N}=3$ quotient, or S-fold. If we take, for instance, the case $p=k=4$, the constraints of the $\rho$ and $\tau$ parameters imply that, out of the five independent radii of $T^{5}$, only one is independent, namely

$$
R_{a}=R_{b}=R_{d}=R_{e}=R, \quad R_{c}=R^{-2} .
$$

The four dimensional theory is reached when $R \rightarrow 0$. 
In appendix $\mathrm{A}$, we describe how the monodromies are embedded in the duality group of M-theory on $T^{5}$, namely $O(5,5 ; \mathbb{Z})$. We use this embedding for computing the supercharges preserved by the non-geometric orbifold

$$
\mathbb{R}^{1,3} \times\left(\mathbb{C} \times T^{5}\right) /\left(\mathbb{Z}_{p}^{E} \times \mathbb{Z}_{k}^{S}\right)
$$

This computation shows that the theory indeed preserves twelve supercharges in four dimensions.

\section{Conclusions}

In this note we have constructed a new set of $\mathcal{N}=3$ SCFTs in four dimensions associated with exceptional algebras. We have no reason to discard the existence of a purely geometric construction of these theories, but the simplest approach, in terms of the (0,2) E-type theories in six-dimensions, led us naturally to consider M-theory on U-manifolds, rather than an ordinary geometric compactification.

Along the way we have encountered a surprise: the action on the M5 brane worldvolume involves a discrete generator which is not part of the usually considered (geometric) $\mathrm{SO}(5)$ R-symmetry group. It should be interesting to see whether use of this extra symmetry can be useful for extending the class of $\mathcal{N}=2$ theories in four dimensions that can be analyzed along the lines of [5]. In particular, it should be enlightening to approach, from this sixdimensional viewpoint, the $\mathcal{N}=3$ theories constructed here and in [11]. A first interesting step would be to reformulate the exotic discrete $\mathbb{Z}_{k}$ action leading to the $\mathcal{N}=4 \rightarrow \mathcal{N}=3$ breaking in terms of the two dimensional theory associated with the four dimensional theory of interest [29]. We hope to report on this topic in the future.

One well known aspect of $(0,2)$ theories compactified on $T^{2}$ is that one can include variants with a non-trivial twisting by an outer automorphism along the $T^{2}$, for instance in order to construct the non-simply laced $\mathcal{N}=4$ theories [30,31], or in order to construct new $\mathcal{N}=2$ theories [32]. We have not considered twisting by such outer automorphisms in this note, but it would be a very interesting direction for further work. In particular, we expect such a construction to describe some or all of the S-fold variants discussed in [11, 22].

Another interesting direction is to generalize the class of compactifications slightly, to include spaces in which the $T^{2}$ factor is fibered non-trivially over the four-dimensional base, while preserving some supersymmetry. This setup appears naturally in the $A_{N-1}$ case, where the resulting $\mathcal{N}=4$ theories with duality defects are useful for understanding aspects of the physics of euclidean D3-branes in F-theory language [33-47]. There is no known corresponding notion of an "exceptional instanton", but the abstract study of the generalization of such duality defects to exceptional theories should be interesting in any case, and the non-geometric backgrounds described in this note give one way of explicitly constructing such setups.

More generally, it is natural to wonder whether non-geometric engineering leads to a richer class of possibilities for constructing six and four dimensional field theories beyond those accessible via geometric techniques, as we suspect to be the case in the particular example we have studied here. Of course, one always ends up having to pay the piper: our 
understanding and control of U-manifolds, particularly their moduli spaces and singularities, is still in its infancy, so currently we can say rather less about this class of theories than about those with geometric constructions. For instance, a basic question about the theories we have constructed that is not straightforward to answer is determining the dimension of their Coulomb branch (for instance, in order to connect, down the road, with the classification program of $[21,48-50])$. The natural object to study would be the set of supersymmetric deformations of the U-manifold we constructed, and to our knowledge there is currently no simple way of approaching this question.

Nevertheless we hope that the existence of the exceptional theories constructed in this note provides good motivation for taking this "non-geometric engineering of QFTs" viewpoint seriously, and developing it further.

\section{Acknowledgments}

We thank Dieter Lüst, Emanuel Malek and Daniel Park for enlightening discussions. D.R thanks the University of Wisconsin-Madison for hospitality. D.R is supported by a grant from the Max Planck Society.

\section{A Details on the $\mathcal{N}=3$ exceptional quotient}

The duality group of M-theory on $T^{5}$ is $O(5,5 ; \mathbb{Z})$. The maximal compact subgroup of the continuous version is $\mathrm{SO}(5) \times \mathrm{SO}(5)$, and the supercharges transform as

$$
\left(S_{6}^{+}, \mathbf{1}, \mathbf{4}\right) \oplus\left(S_{6}^{-}, \mathbf{4}, \mathbf{1}\right)
$$

under $\mathrm{SO}(1,5) \times \mathrm{SO}(5) \times \mathrm{SO}(5)$. We would like to understand how the monodromies of the E-type and S-fold quotients are embedded in $\mathrm{SO}(5) \times \mathrm{SO}(5)$ and how they act on the supercharges. Recall that the 4 of $\mathrm{SO}(5)$ is

$$
\left\{\left(\frac{1}{2} \frac{1}{2}\right),\left(\frac{1}{2}-\frac{1}{2}\right),\left(-\frac{1}{2} \frac{1}{2}\right),\left(-\frac{1}{2}-\frac{1}{2}\right)\right\} \text {. }
$$

In the following we will omit the $\frac{1}{2}$ and simply write $(++)$, etc.

Firstly, there is an $\mathrm{SO}(5)_{S} \subset \mathrm{SO}(5) \times \mathrm{SO}(5)$ that corresponds to the structure group of $T^{5}$, i.e. the geometrical $\mathrm{SO}(5)_{S}$. Clearly, such a subgroup must act in the same way on the supercharges of both chiralities. This can be seen by taking an eleven dimensional spinor and decomposing it according to the splitting $\mathbb{R}^{1,10} \rightarrow \mathbb{R}^{1,5} \times T^{5}$, namely

$$
S_{11}=\left(S_{6}^{+}, \mathbf{4}\right) \oplus\left(S_{6}^{-}, \mathbf{4}\right) .
$$

Thus, from (A.1) and (A.3), we see that $\mathrm{SO}(5)_{S}$ is embedded as

$$
\mathrm{SO}(5)_{S}=\{(g, g) \in \mathrm{SO}(5) \times \mathrm{SO}(5)\},
$$

so it is the diagonal subgroup. We define also the anti-diagonal subgroup

$$
\mathrm{SO}(5)_{A}=\left\{\left(g, g^{-1}\right) \in \mathrm{SO}(5) \times \mathrm{SO}(5)\right\} .
$$


The E-type and S-fold quotients involve geometric rotations in two different tori, $T^{a b}$ and $T^{d e}$, of $T^{5}$. Then, we choose the Cartan subalgebra of $\mathrm{SO}(5) \times \mathrm{SO}(5)$ such that the Cartan of $\mathrm{SO}(5)_{S}$ corresponds to rotations along $T_{a b}^{2}$ and $T_{d e}^{2}$. The quotients also involve non-geometric actions on two three-tori, $T_{a b c}^{3}$ and $T_{c d e}^{3}$, which correspond to the Cartan of $\mathrm{SO}(5)_{A}$.

Example: geometric rotation around $T_{a b}^{2}$. Consider a rotation of $2 \pi / k$ around $T_{a b}^{2}$. The action on the supercharges (A.1) is

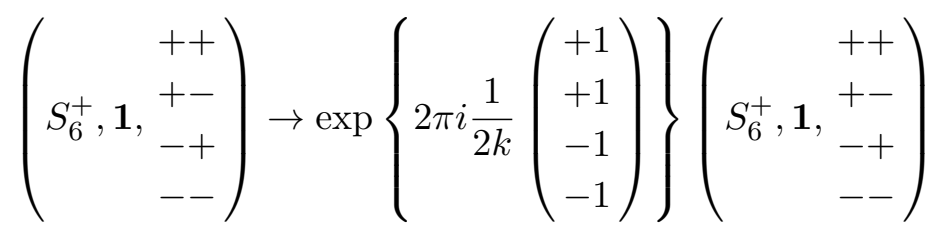

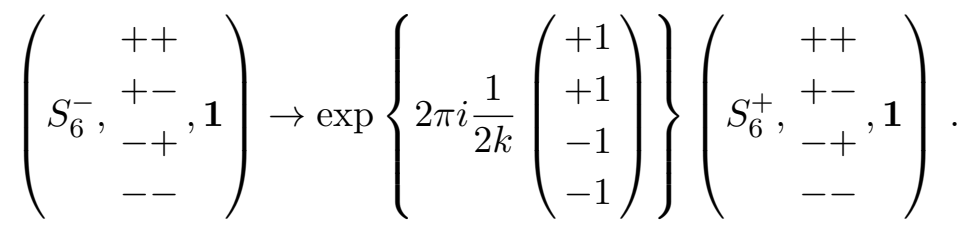

The rotation acts on the first entry of the weight and in the same way for both supercharges.

Example: non-geometric rotation around $T_{a b c}^{2}$. Consider a non-geometric rotation of $2 \pi / k$ around $T_{a b c}^{3}$. The action on the supercharges (A.1) is dictated by the embed$\operatorname{ding}($ A.5) and is

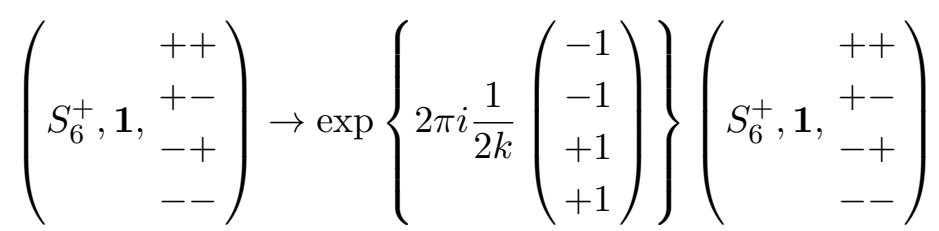

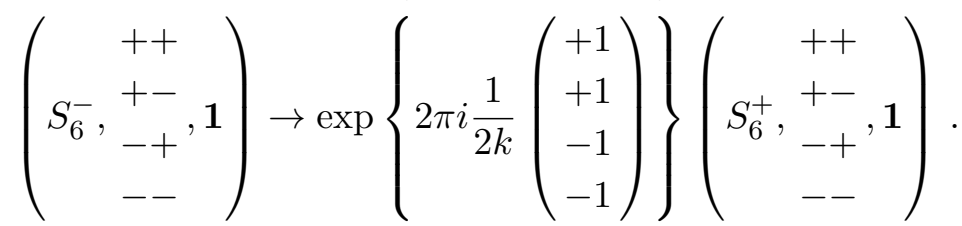

The non-geometric rotation acts on the first entry of the weight and in the opposite way for both supercharges.

Supercharges. The quotient that yields the exceptional $\mathcal{N}=3$ theories is $\mathbb{Z}_{p}^{E} \times \mathbb{Z}_{k}^{S}$, so the supercharges that survive must be invariant under both $\mathbb{Z}_{p}^{E}$ and $\mathbb{Z}_{k}^{S}$ separately. Since these quotients involve an additional $\mathbb{C}$, we split the supercharges in (A.1) as

$$
\left(S_{4}^{+}, \frac{1}{2}, \mathbf{1}, \mathbf{4}\right) \oplus\left(S_{4}^{-},-\frac{1}{2}, \mathbf{1}, \mathbf{4}\right) \oplus\left(S_{4}^{+},-\frac{1}{2}, \mathbf{4}, \mathbf{1}\right) \oplus\left(S_{4}^{-},+\frac{1}{2}, \mathbf{4}, \mathbf{1}\right)
$$

which is how they transform under $\mathrm{SO}(1,3) \times \mathrm{U}(1)_{\mathbb{C}} \times \mathrm{SO}(5) \times \mathrm{SO}(5)$. 
E-type quotient. This consists of a rotation around $\mathbb{C}$ together with a non-geometric rotation around $T_{c d e}^{3}$. The latter acts on the second weight in the opposite way for $(\mathbf{1}, \mathbf{4})$ and $(\mathbf{4}, \mathbf{1})$.

We find

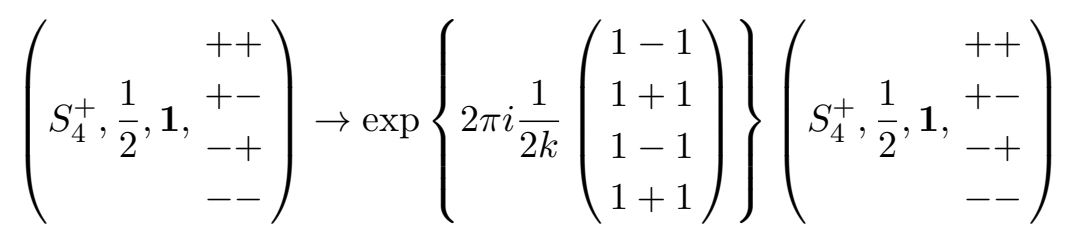

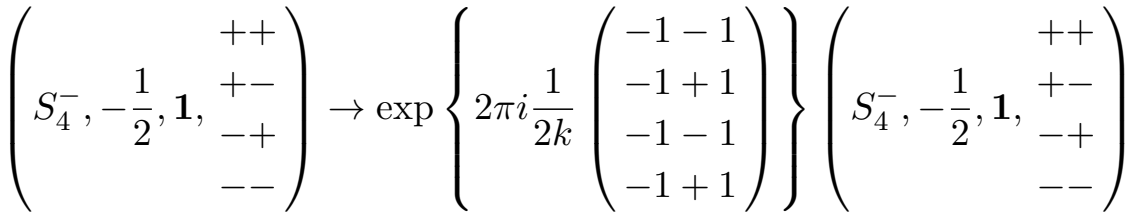

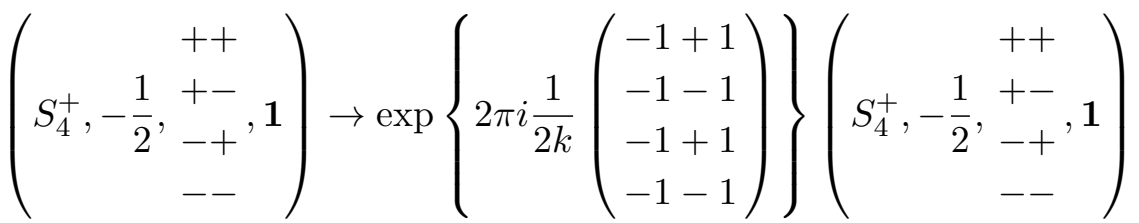

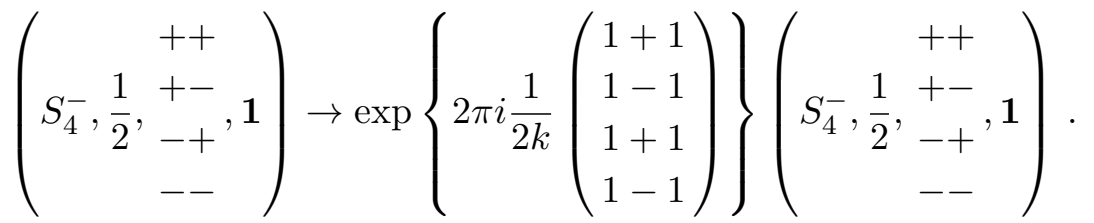

This shows that only half of the supercharges survive, namely

$$
\begin{aligned}
& \left(S_{4}^{+}, \frac{1}{2}, \mathbf{1},++\right) \oplus\left(S_{4}^{+}, \frac{1}{2}, \mathbf{1},-+\right) \oplus\left(S_{4}^{-},-\frac{1}{2}, \mathbf{1},+-\right) \oplus\left(S_{4}^{-},-\frac{1}{2}, \mathbf{1},--\right) \\
& \oplus\left(S_{4}^{+},-\frac{1}{2},++, \mathbf{1}\right) \oplus\left(S_{4}^{+},-\frac{1}{2},-+, \mathbf{1}\right) \oplus\left(S_{4}^{-}, \frac{1}{2},+-, \mathbf{1}\right) \oplus\left(S_{4}^{-}, \frac{1}{2},--, \mathbf{1}\right) .
\end{aligned}
$$

S-fold quotient. In this case we have to take a quotient by a geometric rotation in $\mathbb{C}, T_{a b}^{2}$ and $T_{d e}^{2}$. The last two act on the first and second entries of the weight vector, respectively, and in the same way for $(\mathbf{1}, \mathbf{4})$ and $(\mathbf{4}, \mathbf{1})$. We also need to take a quotient by a non-geometric rotation in $T_{a b c}^{3}$.

The action of this quotient on the supercharges (A.15) that survive the E-type quotient is

$$
\left(S_{4}^{+}, \frac{1}{2}, \mathbf{1},++\right) \rightarrow \exp \left\{2 \pi i \frac{1}{2 k}(1+1-1-1)\right\}\left(S_{4}^{+}, \frac{1}{2}, \mathbf{1},++\right) .
$$

The four contributions to the phase $\exp \left\{2 \pi i \frac{1}{2 k}(1+1-1-1)\right\}$ should be understood as coming, in order, from: 1) rotation in $\mathbb{C}, 2)$ rotation in $\left.T_{a b}^{2}, 3\right)$ rotation in $T_{d e}^{2}$ and 4) 
non-geometric rotation in $T_{a b c}^{3}$. The action on the rest is

$$
\begin{aligned}
\left(S_{4}^{+}, \frac{1}{2}, \mathbf{1},-+\right) & \rightarrow \exp \left\{2 \pi i \frac{1}{2 k}(1-1-1+1)\right\}\left(S_{4}^{+}, \frac{1}{2}, \mathbf{1},-+\right) \\
\left(S_{4}^{-},-\frac{1}{2}, \mathbf{1},+-\right) & \rightarrow \exp \left\{2 \pi i \frac{1}{2 k}(-1+1+1-1)\right\}\left(S_{4}^{-},-\frac{1}{2}, \mathbf{1},+-\right) \\
\left(S_{4}^{-},-\frac{1}{2}, \mathbf{1},--\right) & \rightarrow \exp \left\{2 \pi i \frac{1}{2 k}(-1-1+1+1)\right\}\left(S_{4}^{-},-\frac{1}{2}, \mathbf{1},--\right) \\
\left(S_{4}^{+},-\frac{1}{2},++, \mathbf{1}\right) & \rightarrow \exp \left\{2 \pi i \frac{1}{2 k}(-1+1-1+1)\right\}\left(S_{4}^{+},-\frac{1}{2},++, \mathbf{1}\right) \\
\left(S_{4}^{+},-\frac{1}{2},-+, \mathbf{1}\right) & \rightarrow \exp \left\{2 \pi i \frac{1}{2 k}(-1-1-1-1)\right\}\left(S_{4}^{+},-\frac{1}{2},-+, \mathbf{1}\right) \\
\left(S_{4}^{-}, \frac{1}{2},+-, \mathbf{1}\right) & \rightarrow \exp \left\{2 \pi i \frac{1}{2 k}(1+1+1+1)\right\}\left(S_{4}^{-}, \frac{1}{2},+-, \mathbf{1}\right) \\
\left(S_{4}^{-}, \frac{1}{2},--, \mathbf{1}\right) & \rightarrow \exp \left\{2 \pi i \frac{1}{2 k}(1-1+1-1)\right\}\left(S_{4}^{-}, \frac{1}{2},--, \mathbf{1}\right) .
\end{aligned}
$$

We see that, in total, twelve supercharges survive the action of $\mathbb{Z}_{p}^{E}$ and $\mathbb{Z}_{k}^{S}$. Thus, the quotient $\mathbb{Z}_{p}^{E} \times \mathbb{Z}_{k}^{S}$ yields an $\mathcal{N}=3$ theory in four dimensions.

Open Access. This article is distributed under the terms of the Creative Commons Attribution License (CC-BY 4.0), which permits any use, distribution and reproduction in any medium, provided the original author(s) and source are credited.

\section{References}

[1] N. Seiberg and E. Witten, Electric-magnetic duality, monopole condensation and confinement in $N=2$ supersymmetric Yang-Mills theory, Nucl. Phys. B 426 (1994) 19 [Erratum ibid. B 430 (1994) 485] [hep-th/9407087] [INSPIRE].

[2] N. Seiberg and E. Witten, Monopoles, duality and chiral symmetry breaking in $N=2$ supersymmetric QCD, Nucl. Phys. B 431 (1994) 484 [hep-th/9408099] [INSPIRE].

[3] T. Banks, M.R. Douglas and N. Seiberg, Probing F-theory with branes, Phys. Lett. B 387 (1996) 278 [hep-th/9605199] [INSPIRE].

[4] S.H. Katz, A. Klemm and C. Vafa, Geometric engineering of quantum field theories, Nucl. Phys. B 497 (1997) 173 [hep-th/9609239] [INSPIRE].

[5] D. Gaiotto, $N=2$ dualities, JHEP 08 (2012) 034 [arXiv:0904.2715] [INSPIRE].

[6] Y. Tachikawa, $\mathcal{N}=2$ supersymmetric dynamics for pedestrians, Lecture Notes Phys. 890 (2014) 1 [arXiv: 1312.2684].

[7] A. Kumar and C. Vafa, U manifolds, Phys. Lett. B 396 (1997) 85 [hep-th/9611007] [INSPIRE].

[8] S. Hellerman, J. McGreevy and B. Williams, Geometric constructions of nongeometric string theories, JHEP 01 (2004) 024 [hep-th/0208174] [INSPIRE].

[9] C.M. Hull, A geometry for non-geometric string backgrounds, JHEP 10 (2005) 065 [hep-th/0406102] [INSPIRE]. 
[10] C.M. Hull, Generalised geometry for M-theory, JHEP 07 (2007) 079 [hep-th/0701203] [INSPIRE].

[11] I. García-Etxebarria and D. Regalado, $\mathcal{N}=3$ four dimensional field theories, JHEP 03 (2016) 083 [arXiv: 1512.06434] [INSPIRE].

[12] P.C. Argyres, A. Kapustin and N. Seiberg, On S-duality for non-simply-laced gauge groups, JHEP 06 (2006) 043 [hep-th/0603048] [INSPIRE].

[13] L. Martucci, J.F. Morales and D. Ricci Pacifici, Branes, U-folds and hyperelliptic fibrations, JHEP 01 (2013) 145 [arXiv:1207.6120] [INSPIRE].

[14] A.P. Braun, F. Fucito and J.F. Morales, U-folds as K3 fibrations, JHEP 10 (2013) 154 [arXiv: 1308.0553] [INSPIRE].

[15] P. Candelas, A. Constantin, C. Damian, M. Larfors and J.F. Morales, Type IIB flux vacua from G-theory I, JHEP 02 (2015) 187 [arXiv:1411.4785] [INSPIRE].

[16] P. Candelas, A. Constantin, C. Damian, M. Larfors and J.F. Morales, Type IIB flux vacua from G-theory II, JHEP 02 (2015) 188 [arXiv:1411.4786] [INSPIRE].

[17] A. Font, I. García-Etxebarria, D. Lüst, S. Massai and C. Mayrhofer, Heterotic T-fects, $6 D$ SCFTs and F-theory, JHEP 08 (2016) 175 [arXiv:1603.09361] [INSPIRE].

[18] S. Ferrara, M. Porrati and A. Zaffaroni, $N=6$ supergravity on $A d S_{5}$ and the $\mathrm{SU}(2,2 / 3)$ superconformal correspondence, Lett. Math. Phys. 47 (1999) 255 [hep-th/9810063] [INSPIRE].

[19] O. Aharony and M. Evtikhiev, On four dimensional $N=3$ superconformal theories, JHEP 04 (2016) 040 [arXiv: 1512.03524] [INSPIRE].

[20] T. Nishinaka and Y. Tachikawa, On $4 d$ rank-one $\mathcal{N}=3$ superconformal field theories, JHEP 09 (2016) 116 [arXiv: 1602.01503] [INSPIRE].

[21] P.C. Argyres, M. Lotito, Y. Lü and M. Martone, Expanding the landscape of $\mathcal{N}=2$ rank 1 SCFTs, JHEP 05 (2016) 088 [arXiv: 1602.02764] [INSPIRE].

[22] O. Aharony and Y. Tachikawa, S-folds and $4 d N=3$ superconformal field theories, JHEP 06 (2016) 044 [arXiv: 1602.08638] [INSPIRE].

[23] Y. Imamura and S. Yokoyama, Superconformal index of $\mathcal{N}=3$ orientifold theories, J. Phys. A 49 (2016) 435401 [arXiv: 1603.00851] [inSPIRE].

[24] C. Cordova, T.T. Dumitrescu and K. Intriligator, Deformations of superconformal theories, JHEP 11 (2016) 135 [arXiv: 1602.01217] [INSPIRE].

[25] E. Witten, Some comments on string dynamics, in the proceedings of Future perspectives in string theory (Strings95), March 13-18, Los Angeles, U.S.A. (1995), hep-th/9507121 [INSPIRE].

[26] O. Aharony, String theory dualities from M-theory, Nucl. Phys. B 476 (1996) 470 [hep-th/9604103] [INSPIRE].

[27] E. Witten, On flux quantization in M-theory and the effective action, J. Geom. Phys. 22 (1997) 1 [hep-th/9609122] [INSPIRE].

[28] A. Hanany and B. Kol, On orientifolds, discrete torsion, branes and M-theory, JHEP 06 (2000) 013 [hep-th/0003025] [INSPIRE]. 
[29] L.F. Alday, D. Gaiotto and Y. Tachikawa, Liouville correlation functions from four-dimensional gauge theories, Lett. Math. Phys. 91 (2010) 167 [arXiv:0906.3219] [INSPIRE].

[30] C. Vafa, Geometric origin of Montonen-Olive duality, Adv. Theor. Math. Phys. 1 (1998) 158 [hep-th/9707131] [INSPIRE].

[31] Y. Tachikawa, On S-duality of 5d super Yang-Mills on $S^{1}$, JHEP 11 (2011) 123 [arXiv: 1110.0531] [INSPIRE].

[32] Y. Tachikawa, $N=2$ S-duality via outer-automorphism twists, J. Phys. A 44 (2011) 182001 [arXiv: 1009.0339] [INSPIRE].

[33] E. Witten, Nonperturbative superpotentials in string theory, Nucl. Phys. B 474 (1996) 343 [hep-th/9604030] [INSPIRE].

[34] M. Cvetič, I. Garcia-Etxebarria and R. Richter, Branes and instantons at angles and the F-theory lift of O(1) instantons, AIP Conf. Proc. 1200 (2010) 246 [arXiv:0911.0012] [INSPIRE].

[35] R. Blumenhagen, A. Collinucci and B. Jurke, On instanton effects in F-theory, JHEP 08 (2010) 079 [arXiv: 1002.1894] [INSPIRE].

[36] R. Donagi and M. Wijnholt, MSW instantons, JHEP 06 (2013) 050 [arXiv: 1005.5391] [INSPIRE].

[37] M. Cvetič, I. Garcia-Etxebarria and J. Halverson, On the computation of non-perturbative effective potentials in the string theory landscape: IIB/F-theory perspective, Fortsch. Phys. 59 (2011) 243 [arXiv: 1009.5386] [inSPIRE].

[38] T.W. Grimm, M. Kerstan, E. Palti and T. Weigand, On fluxed instantons and moduli stabilisation in IIB orientifolds and F-theory, Phys. Rev. D 84 (2011) 066001 [arXiv:1105.3193] [INSPIRE].

[39] J. Marsano, N. Saulina and S. Schäfer-Nameki, G-flux, M5 instantons and U(1) symmetries in F-theory, Phys. Rev. D 87 (2013) 066007 [arXiv:1107.1718] [InSPIRE].

[40] M. Cvetič, I. Garcia Etxebarria and J. Halverson, Three looks at instantons in F-theory New insights from anomaly inflow, string junctions and heterotic duality, JHEP 11 (2011) 101 [arXiv: 1107.2388] [INSPIRE].

[41] M. Bianchi, A. Collinucci and L. Martucci, Magnetized E3-brane instantons in F-theory, JHEP 12 (2011) 045 [arXiv:1107.3732] [INSPIRE].

[42] M. Bianchi, A. Collinucci and L. Martucci, Freezing E3-brane instantons with fluxes, Fortsch. Phys. 60 (2012) 914 [arXiv:1202.5045] [INSPIRE].

[43] M. Cvetič, R. Donagi, J. Halverson and J. Marsano, On seven-brane dependent instanton prefactors in F-theory, JHEP 11 (2012) 004 [arXiv:1209.4906] [INSPIRE].

[44] M. Bianchi, G. Inverso and L. Martucci, Brane instantons and fluxes in F-theory, JHEP 07 (2013) 037 [arXiv: 1212.0024] [INSPIRE].

[45] L. Martucci, Topological duality twist and brane instantons in F-theory, JHEP 06 (2014) 180 [arXiv: 1403.2530] [INSPIRE].

[46] A. Gadde, S. Gukov and P. Putrov, Duality defects, arXiv:1404.2929 [INSPIRE].

[47] B. Assel and S. Schäfer-Nameki, Six-dimensional origin of $\mathcal{N}=4 S Y M$ with duality defects, JHEP 12 (2016) 058 [arXiv:1610.03663] [INSPIRE]. 
[48] P. Argyres, M. Lotito, Y. Lü and M. Martone, Geometric constraints on the space of $N=2$ SCFTs I: physical constraints on relevant deformations, arXiv:1505.04814 [INSPIRE].

[49] P.C. Argyres, M. Lotito, Y. Lü and M. Martone, Geometric constraints on the space of $N=2$ SCFTs II: construction of special Kähler geometries and $R G$ flows, arXiv: 1601.00011 [INSPIRE].

[50] P. Argyres, M. Lotito, Y. Lü and M. Martone, Geometric constraints on the space of $N=2$ SCFTs III: enhanced Coulomb branches and central charges, arXiv:1609.04404 [INSPIRE]. 